\title{
CP Puppis: Another V1500 Cyg?
}

\section{O'Donoghue and B. Warncr Dept. of Astronomy, Univ. of Cape Town.}

W. Wargau Dept. of Maths, Appl. Maths and Astronomy, Univ. of South Africa.

A.D. Grauer Dept. of Physics and Astronomy, Univ. of Arkansas at Little Rock.

\section{Introduction}

As first pointed out by Warner (1985), the overall properties of CP Pup and V1500 Cyg are very similar: both were very fast, very large amplitude novae with $\sim 0.3 \mathrm{mag}$ photometric humps at roughly the orbital period. Neither variation is strictly periodic (Patterson 1979; Warner 1985). Variable circular polarization (with period slightly different from the orbital period) has been found in V1500 Cyg showing that its white dwarf is strongly magnetic and has broken synchronism with the secondary star (Stockman, Schmidt \& Lamb 1988). In contrast, no circular polarization has been found in CP Pup. Nonetheless, does the slight difference between the photometric and spectroscopic periods indicate it is also a magnetic system (Warner 1985; Bianchini et al. 1985a)?

New photometric and spectroscopic data for CP Pup are reported which resolve the aliases and confusion in the periods found by Bianchini et al. (1985a,b), Warner (1985) and Duerbeck et al. (1987) and allow an examination of the evidence for its magnetic nature.

\section{Obscrvations}

The new data comprise: (i) 48 hrs of white light high speed photometry obtained during January 1986 and March 1988 at SAAO and Mt. Stromlo; (ii) time-resolved spectra (3600-5400 $)$ during April 1985 and March 1988.

The new photometry shows the $\sim 1.5 \mathrm{hr}$ modulation discovered by Warner (1985), but with a large range in amplitude, from $40 \%$ to invisibility, suggestive of beating of two or more unresolved frequencies. The combined SAAO-Mt. Stromlo data allowed the correct alias to be selected in the power spectrum of the 1988 March data; the same period was found in the 1986 January data. This period was slightly different from the shorter of Warner's (1985) possible periods (Table 1).

Fourier analysis of radial velocities, found by cross-correlation, reveal a single period which is consistent with the period found from the new photometry but NOT consistent with Warner's (1985) photometry (Table 1). The Schneider \& Young (1980) Gaussian mask technique was used to measure the amplitude of the radial velocity curve for different parts of the emission line profile and gave values of $\sim 200 \mathrm{~km} \mathrm{~s}^{-1}$ near the line centres, decreasing to $\sim 120 \mathrm{~km} \mathrm{~s}^{-1}$ in the wings. If the large K-velocity of the line wings $\left(120 \mathrm{~km} \mathrm{~s}^{-1}\right)$ is identical to that of the white dwarf and the secondary star obeys Patterson's (1984) empirical period-secondary mass relation, the maximum white dwarf mass allowed is 0.25 $M_{\odot}$, inconsistent with all theoretical models of nova outbursts (e.g. Kovetz \& Prialnik 1985). This conclusion may be avoided either (i) by increasing the secondary mass by at least a factor of two, incompatible with the view that the secondaries in CVs are undermassive for their size or (ii) postulating that 
the motion of the white dwarf is not given by the emission line wings. There is evidence for the latter in many CVs (e.g. Marsh, Horne \& Shipman 1987).

\section{Is CP Pup magnetic?}

The $\sim 1.5-\mathrm{hr}$ photometric modulation is unlikely to be an orbital hump as in Z Cha or U Gem (which are much less variable in size and shape) but could be: (i) analogous to the superhumps seen in the SU UMa dwarf novae during superoutburst; (ii) heating of the inside face of the secondary as in V1500 Cyg; (iii) due to the varying aspect of an accretion column as in the magnetic CVs. If so, the lack of easily detectable circular polarization or a spectroscopic signature characteristic of the polars implies that the column is more akin to those of the intermediate polars than the polars.

The amplitude modulation and apparent changes in period of the $\sim 1.5-\mathrm{hr}$ modulation are suggestive of the possibility of two or more closely-spaced periods, analogous to Stockman, Schmidt \& Lamb's model for V1500 Cyg.

The spectroscopic information in CP Pup is ambiguous: its spectrum shows high excitation lines $(\mathrm{HeI}+\mathrm{HeII}+\mathrm{CIII} / \mathrm{NIII})$, typical of both magnetic CVs and exnovae. We conclude that we do not yet know whether CP Pup is a magnetic CV.

\section{Refercnees}

Bianchini, A., Friedjung, M. \& Sabbadin, F., 1985a. In: Proceedings of the Frascati Workshop: Multifrequency Behaviour Of Galactic Accreting Sources, p.82, ed. F. Giovanelli.

Bianchini, A., Friedjung, M. \& Sabbadin, F., 1985b. In: Proceedings of the ESA Workshop: Recent Results On Cataclysmic Variables, p.77, ESA SP-236, Noordwijk.

Duerbeck, H.W., Scitter, W.C. \& Duemmler, R., 1987. Mon. Not. R. astr. Soc., 229, 653.

Kovetz, A. \& Prialnik, D., 1985. A strophys. J., 291, 812.

Marsh, T.R., Horne, K. \& Shipman, H.L. 1987. Mon. Not. R. astr. Soc., $225,551$.

Patterson, J., 1979. Astrophys. J., 231, 789.

Patterson, J., 1984. Astrophys. J. Suppl., 54, 443.

Schneider, D.P. \& Young, P., 1980. Astrophys. J., 238, 946.

Stockman, H.S., Schmidt, G.D. \& Lamb, D.Q., 1988. Astrophys. J., 332, 282.

Warner, B., 1985. Mon. Not. R. astr. Soc., 217,1P.

Table 1: Fourier Analysis

\begin{tabular}{|c|c|c|c|}
\hline & $\begin{array}{l}\text { Period } \\
\text { (days) }\end{array}$ & $\begin{array}{l}\text { Mean Semi- } \\
\text { Amplitude }\end{array}$ & $\begin{array}{l}\text { Time of Max. } \\
\text { (HJD) }\end{array}$ \\
\hline $\mathrm{m}, \mathrm{x}$ & & & \\
\hline Warner & 0.06198 & 0.073 & 6112.3446 \\
\hline $1986 \mathrm{Jan}$ & 0.06138 & 0.074 & 6443.4119 \\
\hline 1988 Mar & 0.06154 & 0.071 & 7235.3119 \\
\hline r.: & & $\mathrm{K}\left(\mathrm{km} \mathrm{s} \mathrm{s}^{-1}\right)$ & Sup. Conj. \\
\hline $1985 \mathrm{Apr}$ & $0.06141( \pm 3)$ & $117( \pm 9)$ & $6165.3342( \pm 11)$ \\
\hline 1988 Mar & $0.06148( \pm 5)$ & $121( \pm 7)$ & $7236.4183( \pm 11)$ \\
\hline Duerbeck et al & $0.06143( \pm 3)$ & $92( \pm 18)$ & \\
\hline
\end{tabular}

